\title{
Pleuropulmonary and Lymph Node Progression after Docetaxel - Benefits from Treatment with Cabazitaxel in Metastatic Prostate Cancer
}

\author{
Angel Segura Huerta Encarnación Reche Santos \\ Gema Bruixola Campos Carmen Salvador Coloma Oscar Niño Gómez \\ Medical Oncology Department, Hospital Universitari i Politècnic La Fe, Valencia, Spain
}

\section{Key Words}

Cabazitaxel $\cdot$ Castration-resistant prostate cancer $\cdot$ Pleuropulmonary progression

\begin{abstract}
Introduction: To date, there are no guidelines for a rational and more favourable sequence of treatment after docetaxel. Two drugs (cabazitaxel and abiraterone) have recently been approved as second-line treatment after docetaxel failure in metastatic castration-resistant prostate cancer (mCRPC), but there are no studies comparing abiraterone versus cabazitaxel. The most suitable drug is chosen based on the physician's opinion and the patient's characteristics. In patients with a good performance status who are able to receive either treatment, it would be convenient to begin with cabazitaxel and to reserve abiraterone in case there is a worsening of the general status, in consideration of abiraterone's more favourable toxicity profile. Case Report: We describe the case of a 74-year-old male with mCRPC who presented with an interesting and uncommon tumour dissemination (pleuropulmonary) occurring after the first standard treatment with docetaxel. Intravenous treatment with cabazitaxel $25 \mathrm{mg} / \mathrm{m}^{2}$ and oral prednisone $10 \mathrm{mg}$ continuously was initiated. The patient received a total of 8 cycles of chemotherapy. A reduction of mediastinal adenopathies and infrarenal para-aortic stable bone involvement and an absence of pleural effusion were observed. No relevant toxicity was noted. Since February 2012, a progressive PSA increase without clinical deterioration has been noted. Conclusions: The selection criteria for second- and third-line systemic treatment and the excellent response obtained with cabazitaxel in an unusual disease setting are described. The results confirm the long
\end{abstract}


duration and quality of response of cabazitaxel treatment. Further therapeutic options in this group of patients are suggested.

(C) 2013 S. Karger AG, Basel

\section{Introduction}

Metastatic castration-resistant prostate cancer (MCRPC) has been considered an aggressive and incurable disease with poor prognosis. In the last 10 years, the standard first-line mCRPC treatment has been docetaxel every 3 weeks combined with prednisone [1, 2]. The TROPIC phase III clinical trial confirmed the effectiveness of cabazitaxel versus mitoxantrone in terms of disease survival (15.1 vs. 12.1 months) in patients who had progressed after docetaxel, also conferring an improvement in quality of life [3]. Results from this study led to cabazitaxel approval as second-line treatment in mCRPC. More recently, in 2010, another chemotherapeutic agent, abiraterone, demonstrated improved survival versus placebo (14.8 vs. 10.9 months, respectively) in mCRPC after docetaxel failure (COU-AA-301 phase III study) [4, 5]; abiraterone was also approved for use as second-line treatment after docetaxel failure.

To date, there are no guidelines for a rational and more favourable sequence of treatment after docetaxel, nor are there studies comparing abiraterone versus cabazitaxel. Thus, the most suitable drug is chosen based on the physician's opinion and the patient's characteristics. In case of patients with a good performance status who are able to receive either treatment with cabazitaxel or abiraterone, it would be convenient to begin with the former and to reserve the latter drug in case there is a worsening of the general status, in consideration of the more favourable toxicity profile of abiraterone.

\section{Case Report}

A 74-year-old male with performance status 0 presented with a prostate specific antigen (PSA) level of $51.2 \mathrm{ng} / \mathrm{ml}$ in August 2001. After biopsy, computed axial tomography (CT) and bone scan, prostate adenocarcinoma T2bN0M0, Gleason $6(2+4)$, was diagnosed and considered an intermediate risk. The patient was treated with radical prostate-bed radiotherapy and full androgenic block with oral (PO) bicalutamide $50 \mathrm{mg} / 24 \mathrm{~h}$ and subcutaneous goserelin $10.8 \mathrm{mg}$ quarterly until March 2002, when it was decided to remove the blockade after reaching an almost undetectable PSA level.

He was asymptomatic with a stable PSA level less than $0.07 \mathrm{ng} / \mathrm{ml}$ until March 2003, when the PSA level started increasing until reaching its highest level $(7.27 \mathrm{ng} / \mathrm{ml})$ in May 2005. Because of this biochemical relapse, antiandrogenic treatment was initiated with PO tamoxifen $10 \mathrm{mg} / 12 \mathrm{~h}$ and PO bicalutamide $50 \mathrm{mg} / 24 \mathrm{~h}$, progressively reducing the PSA level (reaching its nadir of $0.66 \mathrm{ng} / \mathrm{ml}$ in September 2006). The blockade was suspended and a quarterly subcutaneous treatment with leuprorelin acetate was initiated. In September 2009, the PSA level rose to $47.53 \mathrm{ng} / \mathrm{ml}$ along with a clinical picture of weakness of the lower limbs, ambulatory difficulty and mechanical lumbar pain.

A thoracic-abdominal-pelvic CT scan was requested; it focused on adenopathies between 1 and $2 \mathrm{~cm}$ in the upper and middle mediastinum and the left infrarenal para-aortics, as well as diffuse bone lesions in the lumbar vertebral bodies. Abnormal deposits of diffuseshaped tracer were detected in the bone scan. Magnetic resonance imaging showed a mass of soft tissue at the spinal level causing a spinal stenosis, maximum T7 level, without compromising the spinal cord, so it was treated with palliative intent radiotherapy (fig. 1). 
Due to the hormonal refractoriness, first-line chemotherapy treatment was started with intravenous docetaxel $75 \mathrm{mg} / \mathrm{m}^{2}$ and PO prednisone $5 \mathrm{mg} / 12 \mathrm{~h}$. The first cycle was given on October 21, 2009. The patient's PSA level at this time was $47.53 \mathrm{ng} / \mathrm{ml}$. He completed 9 cycles of treatment (the last in April 2010) with excellent tolerance. His PSA analytical nadir reached $19.81 \mathrm{ng} / \mathrm{ml}$ after chemotherapy was completed. A further evaluation in May 2010 gave a partial response, continuing only with luteinizing hormone-releasing hormone analogues.

In April 2011, pulmonary progression with right pleural effusion and pathological size increase in paratracheal adenopathies and the aortopulmonary window were observed on a CT scan and histologically confirmed. Due to this situation and the good overall condition of the patient, it was decided to initiate intravenous treatment with cabazitaxel $25 \mathrm{mg} / \mathrm{m}^{2}$ and PO prednisone $10 \mathrm{mg}$ continuously. At the beginning of the treatment, his PSA level was $120.62 \mathrm{ng} / \mathrm{ml}$, reaching $64 \mathrm{ng} / \mathrm{ml}$ after the third cycle in June 2011.

After 6 cycles of chemotherapy, a reduction of mediastinal adenopathies and infrarenal para-aortic stable bone involvement and an absence of pleural effusion were observed. The patient's PSA level was $48.58 \mathrm{ng} / \mathrm{ml}$. Given the good response and the absence of toxicity, it was decided to continue with 2 more cycles. After 8 cycles, the last of which was in September 2011, treatment was discontinued as it was considered that the therapeutic plan had been fulfilled without having relevant toxicity at any time.

In February 2012, his PSA level increased to $94 \mathrm{ng} / \mathrm{ml}$. A thoracic-abdominal-pelvic CT scan was requested, which showed stability of the infradiaphragmatic and supradiaphragmatic gland injuries, as well as diffuse bone metastasis. To date, the patient remains asymptomatic. Since February 2012, a progressive PSA increase without clinical deterioration has been noted. In January 2013, the PSA level was $191 \mathrm{ng} / \mathrm{ml}$ (fig. 2). Should further symptoms occur, a new treatment will be proposed. The reintroduction of cabazitaxel treatment would be our first choice given the clinical benefit and the important targeted radiological and biochemical responses. Currently, there is a treatment-free interval following the end of the 16-month cabazitaxel treatment and a 6-month period free of biological progression.

\section{Discussion}

mCRPC has historically been considered an aggressive and ultimately incurable disease with a poor prognosis, although its treatment has progressed significantly in recent decades. Currently, docetaxel every 3 weeks alongside prednisone is the reference first-line treatment of mCRPC $[1,2]$.

The use of bisphosphonates, in particular zoledronic acid, in patients with prostate cancer and metastatic bone disease has not proven to be superior to the best support treatment in terms of a decrease in bone events such as pathological fractures or spinal cord compressions [6-8]. In our case, we did not use bisphosphonates, considering the good pain control achieved with nonsteroidal anti-inflammatory medicines and transdermal fentanyl.

The evidence from cases in which there is progress after first-line treatment with taxanes has promoted the study and emergence of various agents directed against other therapeutic targets. In this regard, chemotherapy with cabazitaxel, a semisynthetic antimicrotubule agent derived from taxanes, as well as treatment with the inhibitor of cytochrome $\mathrm{P}$, abiraterone acetate, has offered an increase in survival while maintaining a good quality of life. For the first time in the TROPIC phase III clinical trial, the effectiveness of cabazitaxel versus mitoxantrone was proven in terms of survival, also conferring an 
improvement in the quality of life [3]. However, as with other taxanes, it is worth noting that there is haematological toxicity, in particular neutropenia; therefore, administering cabazitaxel in combination with granulocyte colony-stimulating factor is recommended. Moreover, its safety in elderly patients was proven in an interim analysis in 20 countries of the European Union [9]. In our 74-year-old patient, granulocyte colony-stimulating factor was prophylactically administered from the second cycle of cabazitaxel, maintaining optimal levels of the three haematological series during the treatment and showing an excellent tolerance towards cabazitaxel treatment.

This case was atypical due to the presence of pleuropulmonary metastases secondary to prostate cancer, and because of the good subjective and objective response to the cabazitaxel treatment, supporting the results of the TROPIC phase III clinical trial. The presence of visceral metastasis, which defined the disease as aggressive, along with haemoglobin levels and performance status among other aspects were studied in this trial as forecast factors, with the PSA level being unequivocally the most important $[3,7,10]$.

One of the biggest controversies focuses on determining the ideal time and best results of post-docetaxel castration-resistant prostate cancer treatment [11]. Currently, there are no guidelines or protocols indicating which are the most beneficial and logical sequences; there are also no studies which directly compare abiraterone and cabazitaxel. The most suitable drug must be chosen based on the treating physician's judgment and the personal characteristics of the patient.

It seems logical that, in the case of patients with a good performance status capable of receiving treatment both with cabazitaxel and abiraterone, it would be convenient to start with the former, keeping the latter in case of an eventual overall deterioration, acting consistently with the most favourable toxicity profile of abiraterone.

For those who have progressed to docetaxel during mCRPC treatment, a reintroduction of treatment could also be considered if there was a good initial response to first-line treatment (lasting over 4 months), if relapse occurred after 6 or more months after the last cycle, if docetaxel was interrupted because of toxicity, or if a tenth cycle had been reached. Currently, there are no randomized studies that prove a benefit from treatment reintroduction. There are only retrospective studies that do not demonstrate improvement in overall survival. In addition, there is recent data about a probable lower efficacy in the reintroduction of treatment with docetaxel following abiraterone, suggesting some mechanism of cross-resistance [12].

All of the above, along with the existence of drugs such as abiraterone and cabazitaxel which have a favourable response rate and toxicity profile, means that reintroduction of treatment with docetaxel is unusual in everyday medical practice.

\section{Conclusion}

Emphasis in the handling of mCRPC should focus on the chronology of the metastatic disease, with first-line treatment with docetaxel and a long-term vision to maximize the opportunity for patients to receive all or most of the treatments available today, with continuous treatment and the discovery of new agents being as important as their rational and sequential use. In this regard, after progressing to taxanes, treatment with cabazitaxel has shown benefits in overall survival in terms of elderly patients and aggressive disease, along with an acceptable toxicity profile, turning it into the standard MCRPC therapy after progressing to docetaxel [13]. 
Segura Huerta et al.: Pleuropulmonary and Lymph Node Progression after Docetaxel Benefits from Treatment with Cabazitaxel in Metastatic Prostate Cancer

\section{Disclosure Statement}

The authors have no conflicts of interest to declare.

\section{References}

-1 Smaletz 0, Scher HI, Small EJ, et al: Nomogram for overall survival of patients with progressive metastatic prostate cancer after castration. J Clin Oncol 2002;20:3972-3982.

2 Weinfurt KP, Anstrom KJ, Castel LD, et al: Effect of zoledronic acid on pain associated with bone metastasis in patients with prostate cancer. Ann Oncol 2006;17:986.

-3 de Bono JS, Oudard S, Ozguroglu M, et al: Prednisone plus cabazitaxel or mitoxantrone for metastatic castration-resistant prostate cancer progressing after docetaxel treatment: a randomized open-label trial. Lancet 2010;376:1147-1154.

-4 O'Donell A, Judson I, Dowsett M, et al: Hormonal impact of the 17 alpha-hydroxilase/C(17,20)-lyase inhibitor abiraterone acetate (CB7630) in patients with prostate cancer. Br J Cancer 2004;90:2317-2325.

$\checkmark 5$ de Bono JS, Logothetis CJ, Molina A; and COU-AA-301 Investigators: Abiraterone and increased survival in metastatic prostate cancer. Engl J Med 2011;364:1995.

-6 Saad F, Gleason DM, Murray R, et al: A randomized, placebo-controlled trial of zoledronic acid in patients with hormone-refractory metastatic prostate carcinoma. Natl Cancer Inst 2002;94:1458-1468.

7 Basch EM, Somerfield MR, Beer TM, et al: American Society of Clinical Oncology endorsement of the Cancer Care Ontario Practice Guideline on nonhormonal therapy for men with metastatic hormone-refractory (castration-resistant) prostate cancer. J Clin Oncol 2007;25:5313.

-8 Heidenreich A, Hofmann R, Engelmann UH: The use of bisphosphonate for the palliative treatment of painful bone metastasis due to hormone refractory prostate cancer. J Urol 2001;165:136.

9 Heidenreich A, Bracarda S, Mason M, et al: Tolerability of cabazitaxel in senior adults with metastatic castration-resistant prostate cancer (MCRPC) in Europe. 37th ESMO Congress, Vienna, 2012.

$\$ 10$ Hussain M, Goldman B, Tangen C, al: Prostate-specific antigen progression predicts overall survival in patients with metastatic prostate cancer: data from Southwest Oncology Group Trials 9346 (Intergroup Study 0162) and 9916. J Clin Oncol 2009;27:2450-2456.

$\checkmark 11$ Halabi S, Vogelzang NJ, Ou SS, et al: Progression-free survival as a predictor of overall survival in men with castrate-resistant prostate cancer. J Clin Oncol 2009;27:2766-2771.

$\checkmark 12$ Ansari J, Hussain SA, Zarkar A, et al: Docetaxel chemotherapy for metastatic hormone refractory prostate cancer as first-line palliative chemotherapy and subsequent re-treatment: Birmingham experience. Oncol Rep 2008;20:891-896.

13 Sonpavde G, Sternberg CN: Contemporary management of metastatic castration-resistant prostate cancer. Curr Opin Urol 2011;21:241-247. 
Segura Huerta et al.: Pleuropulmonary and Lymph Node Progression after Docetaxel Benefits from Treatment with Cabazitaxel in Metastatic Prostate Cancer

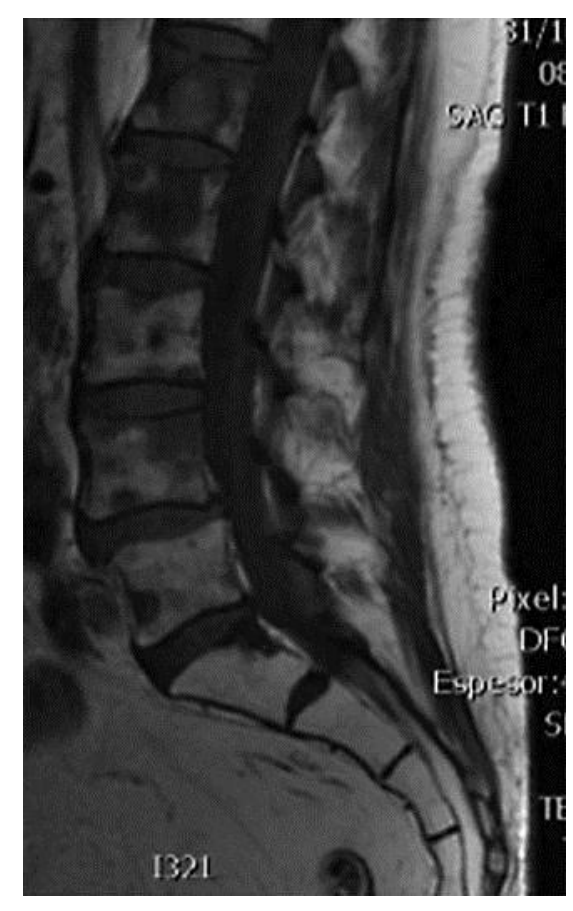

Fig. 1. Metastasis affecting multiple vertebral bodies.

\section{TOTAL PSA}

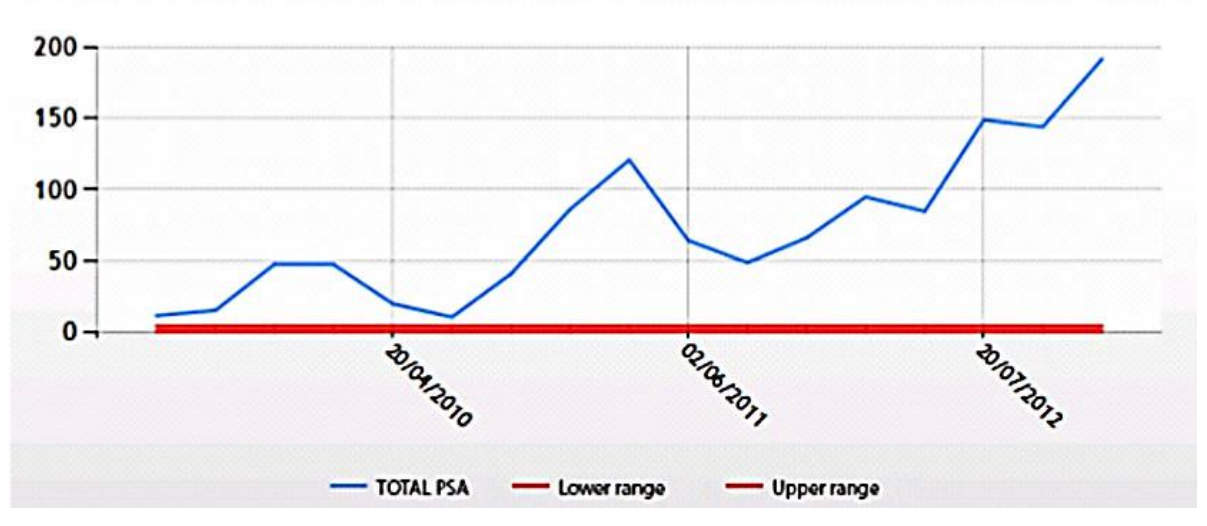

Fig. 2. Evolution of PSA levels. 